\title{
Perfil Farmacológico de Pacientes Hipertensos Cadastrados em uma Unidade de Saúde da Família no Município de Nova Canaã-BA
}

\author{
Jaqueline Ferraz Rodrigues Coqueiro ${ }^{\text {; }}$ Lucy Matos Santos ${ }^{2}$
}

Resumo: De acordo com o Ministério da Saúde, a Hipertensão Arterial Sistêmica (HAS) afeta 600 milhões de pessoas no mundo, cerca de $25 \%$ da população brasileira adulta apresenta hipertensão e na terceira idade aumenta para 50\%. A HAS é caracterizada como um estado clínico que envolve múltiplos fatores e elevado nível da Pressão Arterial (PA), associada a alterações na estrutura e funcionamento de órgãos-alvo (encéfalo, coração, rins e vasos sanguíneos), aumentando assim os riscos de acidentes cardiovasculares que podem levar ou não a óbito o indivíduo. O tratamento farmacológico tem como finalidade primordial, evitar a morbidade e diminuir a mortalidade por eventos cardiovasculares relacionados à HAS. Considerando esses dados o presente estudo tem como objetivo conhecer o perfil farmacológico de pacientes hipertensos cadastrados na Unidade de Saúde da Família no Município de Nova Canaã-BA, através de um questionário que envolve a idade, medicamento, tempo de uso e as queixas relatadas pelo paciente.

Palavras-chave: Hipertensão Arterial Sistêmica. Perfil Farmacológico. Tratamento

\section{Pharmacological profile of hypertensive patients enrolled in a Family Health Unit in the Municipality of Nova Canaã-BA}

\begin{abstract}
According to the Ministry of Health, Systemic Arterial Hypertension (SAH) affects 600 million people worldwide, about $25 \%$ of the Brazilian population has hypertension and in the third age increases to $50 \%$. SAH is characterized as a clinical condition involving multiple factors and a high level of Blood Pressure BP, associated with changes in the structure and functioning of target organs (brain, heart, kidneys and blood vessels), thus increasing the risk of accidents which may or may not lead to death. Pharmacological treatment has the primary purpose of avoiding morbidity and reducing mortality due to cardiovascular events related to hypertension. Considering these data the present study aims to know the pharmacological profile of hypertensive patients enrolled in the Family Health Unit in the Municipality of Nova Canaã-BA, through a questionnaire that involves age, medication, time of use and complaints reported by the patient.
\end{abstract}

Keywords: Hypertension. Pharmacological profile. Treatment.

\footnotetext{
${ }^{1}$ Graduação em Farmácia pela Universidade Tiradentes - UNIT, Mestre em Planejamento Ambiental pela Universidade Católica do Salvador - UCSAL, atualmente é Coordenadora e docente do Curso de Farmácia na Faculdade Independente do Nordeste - FAINOR. Contato: jaquelineferraz@yahoo.com.br.

${ }^{2}$ Graduação em Farmácia pela Farmácia na Faculdade Independente do Nordeste - FAINOR, Email: lucymatos12@hotmail.com
} 


\title{
Introdução
}

De acordo com a Organização Mundial de Saúde - OMS, A Hipertensão Arte Sistêmica - HAS, em média afeta cerca de 600 milhões de pessoas no mundo, no Brasil 25\% da população adulta apresenta hipertensão e na terceira idade esse número aumenta para 50\%. Sendo responsável por $40 \%$ dos infartos, $80 \%$ dos derrames e $25 \%$ dos casos de insuficiência renal terminal (SOUZA et al, 2014).

A HAS é uma doença de evolução lenta e detecção tardia, sendo caracterizada como um dos principais fatores de risco para o desenvolvimento de doenças cardiovasculares e cerebrovasculares, considerada um importante problema que atinge a saúde pública no Brasil (SANTOS, 2011).

Segundo a Sociedade Brasileira de Cardiologia, 2010, a HAS é definida como:

\begin{abstract}
A hipertensão arterial sistêmica (HAS) é uma condição clínica multifatorial caracterizada por níveis elevados e sustentados de pressão arterial - PA (PA $\geq 140 \mathrm{x}$ $90 \mathrm{mmHg}$ ). Associa-se, frequentemente, às alterações funcionais e/ou estruturais dos órgãos-alvo (coração, encéfalo, rins e vasos sanguíneos) e às alterações metabólicas, com aumento do risco de eventos cardiovasculares fatais e não fatais (SOCIEDADE BRASILEIRA DE CARDIOLOGIA, 2010, p. 1).
\end{abstract}

São diversos os fatores e causas que envolvem a Hipertensão Arterial Sistêmica destacando-se as principais: sexo, idade, raça/cor, sedentarismo, obesidade, fatores genéticos, estresse, tabagismo, alcoolismo, alimentação e estilo de vida inadequado (NOBRE et al, 2014).

A hipertensão é um conjunto de sintomas que envolvem fatores genéticos, ambientais, vasculares, hormonais, renais e neurais. O fator genético e o ambiental estão relacionados diretamente com o desenvolvimento da hipertensão, pois o individuo com histórico familiar de hipertensão e se expõe a fatores ambientais ocorre uma desarmonia entre o sistema nervoso simpático com renina-angiotensina gerando a elevação da resistência vascular sistêmica (NOBRE et al, 2014).

Os fatores vasculares estão relacionados com a pressão arterial (PA) através da hemodinâmica, pois a PA é estabelecida pelo débito cardíaco x resistência vascular periférica total, dessa forma se houver o desequilíbrio de um ou de ambos os fatores pode se manifestar a hipertensão (NOBRE et al, 2012). 
O tratamento farmacológico da HAS tem como meta primordial diminuir a morbimortalidade. Dessa forma os anti-hipertensivos além de reduzir os níveis da pressão arterial reduz o risco de eventos cardiovasculares. Os anti-hipertensivos são divididos em distintas categorias de acordo com os diferentes mecanismos que interferem na fisiopatologia da hipertensão (RIBEIRO et al, 2012).

Estudos feitos pela Associação Brasileira de Cardiologia (2007) mostram que o aumento da pressão arterial traz consequências desastrosas nos órgãos e sistema do corpo. $\mathrm{O}$ estudo em questão relata ainda que $40 \%$ das mortes estão associadas a acidente vascular cerebral e $25 \%$ por doenças coronarianas. Esses dados também são apontados por Brasil (2006), que acrescenta outras patologias tais como: doença renal crônica e doença arterial periférica (ÁVILA, 2010).

No Brasil ainda é desconhecido a prevalência da Hipertensão Arterial no país como um todo, há estudos que demonstram apenas a prevalência por regiões isoladas, cerca de 30\% dos indivíduos adultos apresentam HAS, uma revisão sistemática quantitativa mostrou um predomínio mundial de 37,8\% em homens e 32,1\% em mulheres (NOBRE et al, 2013).

Segundo Santos (2011), se a HAS não for tratada, e não houver elaboração de programas para o controle e prevenção dos fatores de risco relacionados com a hipertensão, em 2020 vai ter um aumento no número de pessoas com hipertensão com idade acima dos 60 .

De acordo com informações coletadas no Sistema de Informação de Atenção Básica, no município de Nova Canaã-Ba estão cadastrados 1.381pacientes que apresentam HAS, sendo que 712 desses pacientes são do sexo masculino e 669 do sexo feminino (NOVA CANÃ̃, 2015).

Com base nesses pressupostos, a presente pesquisa delimita-se ao seguinte problema: Qual o perfil farmacológico de pacientes hipertensos cadastrados na unidade de saúde da família no município de Nova Canaã-BA?

Sendo assim, a presente pesquisa tem como objetivo geral conhecer o perfil farmacológico dos pacientes cadastrados na unidade de saúde e como objetivos específicos identificar as principais queixas relatadas pelos pacientes hipertensos e se as mesmas tem alguma relação com o efeito adverso do medicamento em uso e o papel do farmacêutico na equipe de saúde no tratamento e acompanhamento do cliente com HAS. 
O presente estudo será de grande relevância para os pacientes cadastrados na unidade e para os profissionais de saúde, pois será ampliado o leque de informações sobre o tratamento e as queixas relatadas de cada cliente bem como a relação queixa $\mathrm{x}$ reações adversas do medicamento contribuindo assim para prevenção, redução e adesão terapêutica dos pacientes hipertensos.

\section{Metodologia}

Para a elaboração desse projeto foi realizada pesquisa na internet, através de artigos, revistas, manuais e diretrizes, e descritores tais como: Hipertensão Arterial Sistêmica, perfil farmacológico e tratamento.

O estudo em questão é do tipo descritivo-quantitativo, pois os fatos foram analisados e registrados, munidos de técnica de coleta de dados a partir dos prontuários e relato dos pacientes.

O local dessa pesquisa foi em uma Unidade de Saúde da Família Valdemar Andrade, localizado na Rua Jesiel Norberto da Silva, S/N, Centro no município de Nova Canaã-Ba. Esse local foi escolhido, pois oferece acesso e oportunidade de coletar os dados do público alvo com idade e características pertinentes a pesquisa.

A população da pesquisa foi representada por 34 pacientes incluindo sexo masculino e feminino com a faixa etária de 40 a 60 anos sem restrição do grau de escolaridade e estado civil cadastrados no Programa de Hiperdia da referida unidade.

A coleta foi realizada no período de outubro de 2016, logo após a provação do projeto que foi submetido ao Comitê de Ética e Pesquisa (CEP) da instituição de ensino com o protocolo CAAE n60344616.3.0000.5578, e todos os pacientes incluídos assinaram o Termo de Consentimento Livre e Esclarecido.

A coleta foi realizada através de um questionário (Apêndice A), a partir das informações do prontuário dos pacientes cadastrados na unidade, foram coletados cinco itens essenciais para a pesquisa produzida pela própria pesquisadora com a finalidade de tabular os dados com técnicas estatísticas. 
Após a coleta dos dados, os dados obtido foi organizado de forma que cada um foi submetido a uma seleção a fim de inspecionar falhas. Em seguida, os dados foram tabulados e codificados utilizando como auxilio o programa Microsoft Office Excel $2010^{\circledR}$ para o tratamento estatístico, e foi apresentado em forma de gráfico e porcentagem.

\section{Resultados e Discussão}

Para esse estudo foram entrevistados 34 pacientes hipertensos cadastrados na Unidade de Saúde da Família Valdemar Andrade, localizado a Rua Jesiel Norberto da Silva, S/n, Centro no município de Nova Canaã-Ba, na área 001 e micro área 020. Os dados foram coletados através de uma entrevista com a população alvo a partir de um questionário contendo cinco perguntas e os resultados tabulados e expressos a seguir.

O gráfico 1 apresenta a idade dos pacientes com HAS cadastradas na Unidade de Saúde da Família no município de Nova Canaã- BA, pela pesquisa realizada os pacientes apresenta idade entre 40 e 60 anos, sendo a maior predominância com 56 anos que corresponde a 35\% dos pacientes entrevistados.

Segundo ANDRADE et al (2014), no intervalo entre 2006 e 2011 o número de adultos com hipertensão em 2006 atingiu 21,5\% e em 2009 esse número elevou para 34,4\% tendo um crescimento gradativo da HAS com o avanço da idade e ele afirma ainda que cerca da metade dos indivíduos com a faixa etária acima dos 55 anos são mais acometidos.

Gráfico 1- Idade dos pacientes com HAS cadastradas na Unidade de Saúde da Família no município de Nova Canaã-BA.

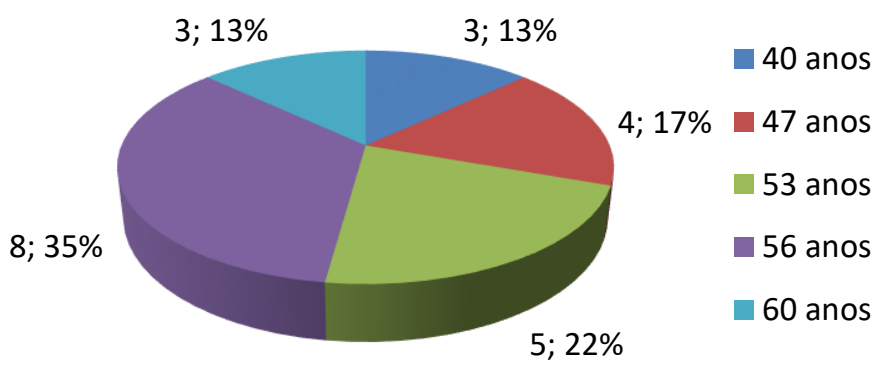

Fonte: Pesquisa de campo, Nova Canaã-BA, 2016. 
De acordo com os dados coletados dos 34 pacientes entrevistados foi visto que 22 são do sexo feminino, corresponde a $65 \%$ e 12 são do sexo masculino que corresponde $35 \%$ da população em estudo, conforme o Gráfico 2.

Apesar de estudos demonstrarem que os homens são mais acometidos pela hipertensão que as mulheres, nos últimos anos esse número tem se igualado, pois as mulheres depois da menopausa sofre um aumento no número de casos devido o declínio dos hormônios sexuais, já que no período fértil esses hormônios apresentam ação favorável para o sistema cardiovascular (OLIVEIRA et al, 2013).

Gráfico 2- Sexo dos pacientes com HAS cadastrados na Unidade de Saúde da Família no município de Nova Canaã-BA.

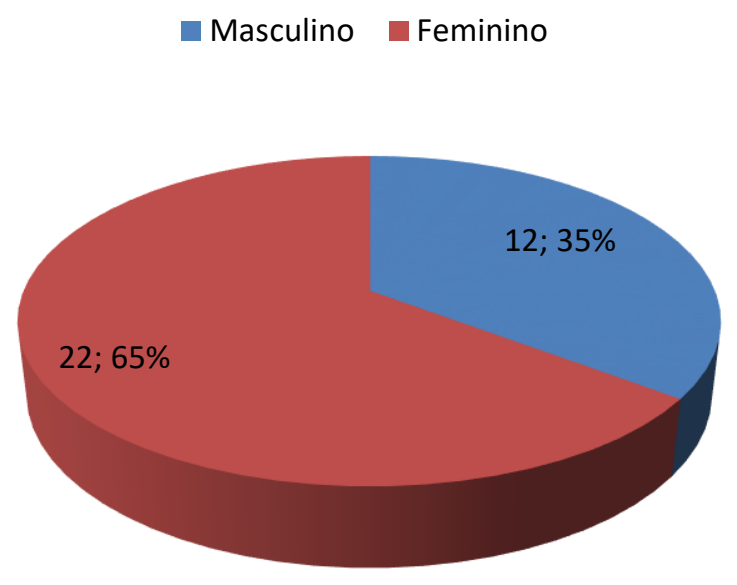

Fonte: Pesquisa campo, Nova Canaã- BA, 2016.

A partir dos dados obtidos, pode-se inferir que o fator socioeconômico da maioria dos pacientes é de 1 a 2 salários mínimos (Gráfico 3). Segundo Machado et al (2010), as condições socioeconômicas tem relação direta com o desenvolvimento da HAS, pois quanto menor a renda familiar menor será o grau de conhecimento sobre a doença e consequentemente o aumento da não adesão terapêutica por não ter acesso ao medicamento. 
Gráfico 3- Fator socioeconômico de pacientes com HAS cadastrados na Unidade de Saúde da Família no município de Nova Canaã- BA.

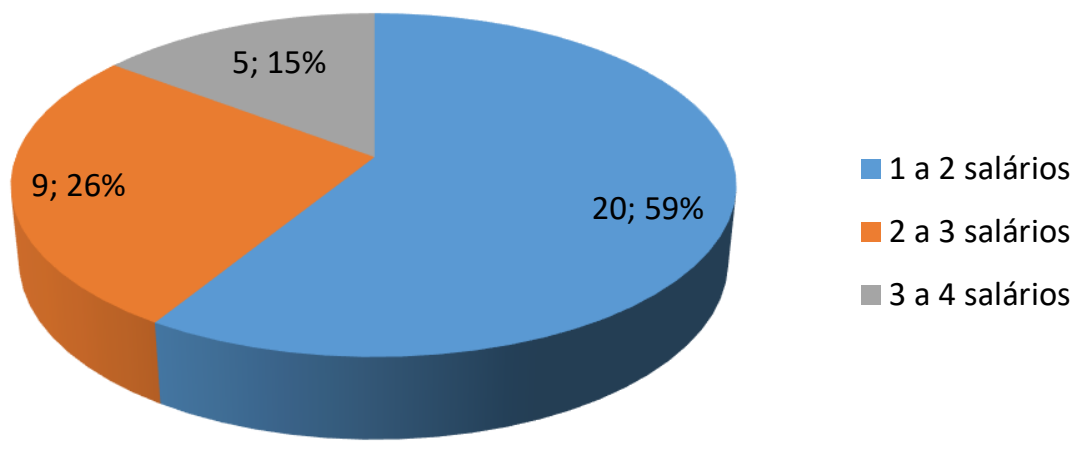

Fonte: Pesquisa de campo, Nova Canaã-BA, 2016.

Dentre os anti-hipertensivos mais utilizados pela população do estudo, a maioria faz uso do Losartana 50mg (bloqueador de receptores AT1), e o Hidroclotiazida 25mg (duréticotiazídico), Enalapril 5mg (inibidor da enzima conversora da angiotensina- ECA), Anlodipino 5mg (bloqueador dos canais de cálcio), Atenolol 25mg (betabloqueador adrenégico).

No tratamento inicial da HAS, para indivíduos que apresentam risco de eventos cardiovasculares a terapia é feita com apenas um anti-hipertensivo, podendo ser um diurético, betabloqueadores, inibidores da ECA e bloqueadores do receptor AT1, ajustando as doses até que a PA atinja o nível ideal (BRASIL, 2010).

De acordo com Brasil (2010), a maioria dos casos a monoterapia não apresenta resultados satisfatórios, sendo necessária a adição de dois ou mais medicamentos de classes e mecanismo de ação diferente. 
Id on Line Revista Multidisciplinar e de Psicoloqia

Id on Line Multidisciplinary and Psycology Journal

Gráfico 4 - Anti-hipertensivos utilizados pelos pacientes com HAS cadastrados na Unidade de Saúde da Família do município de Nova Canaã-BA.

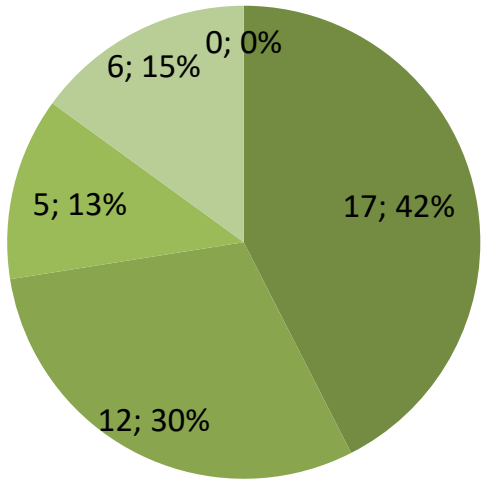

$$
\begin{aligned}
& \text { Losartana } \\
& \text { Hidroclotiazida } \\
& \text { Inalapril } \\
& \text { Anlodipino } \\
& \text { Atenolol }
\end{aligned}
$$

Fonte: Pesquisa de campo, Nova Canaã-BA, 2016.

Através dos dados coletados na pesquisa quanto ao tempo de uso dos anti-hipertensivos a maioria dos pacientes já utiliza o medicamentode 12 a 26 anos e o menor tempo de uso foi de 3 a 11 anos, conforme o Gráfico 5.

Com o uso prolongado dosanti-hipertensivos os pacientes apresentam manifestações clinicas, sendo elas responsáveis pela não adesão ao tratamento como exemplo pode citar a tontura levando o paciente acreditar que os níveis de PA estão baixos e que não necessitam fazer mais o uso do medicamento (TAVARES et al, 2013).

Gráfico 5 - Tempo de uso dos anti-hipertensivos utilizados pelos pacientes com HAS cadastrados na Unidade de Saúde do município de Nova Canaã-BA.

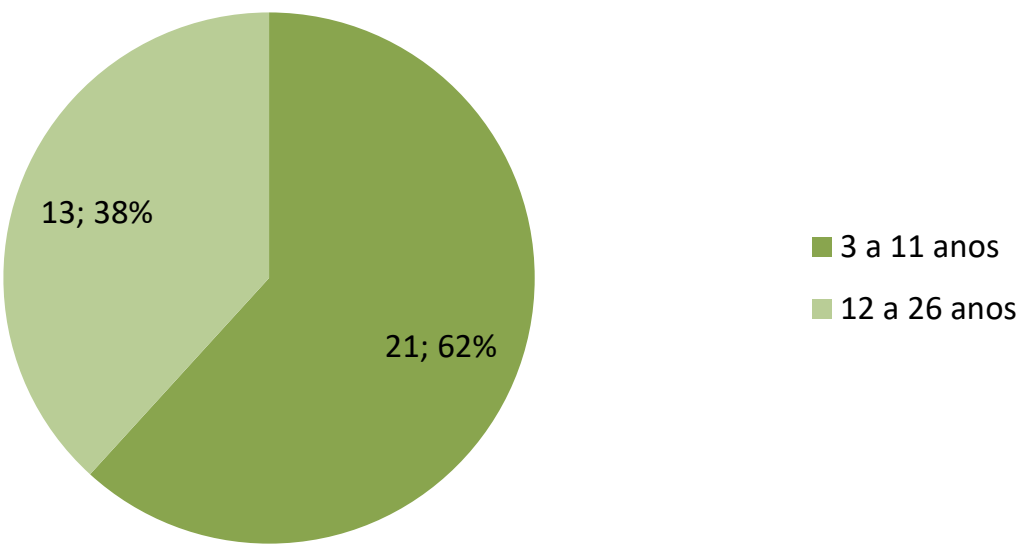

Fonte: Pesquisa de campo, Nova Canaã-BA, 2016 
Pelos dados coletados foi verificado que a maioria dos pacientes não apresentou nenhuma queixa e os pacientes que relataram alguma: cefaleia e dor abdominal (Gráfico 6).

Com relação às queixas apresentadas no estudo a cefaleia é uma reação adversa da classe dos antagonistas dos canais de cálcio, como por exemplo, o Anlodipino, já o desconforto abdominal é uma reação adversa dos antagonistas do receptor da angiotensia II, como exemplo a Losartana (KOROLKOVAS et al, 2010; 2011).

Gráfico 6 - Principais queixas apresentadas pelos pacientes com HAS cadastrados na Unidade de Saúde da Família do município de Nova Canaã-BA.

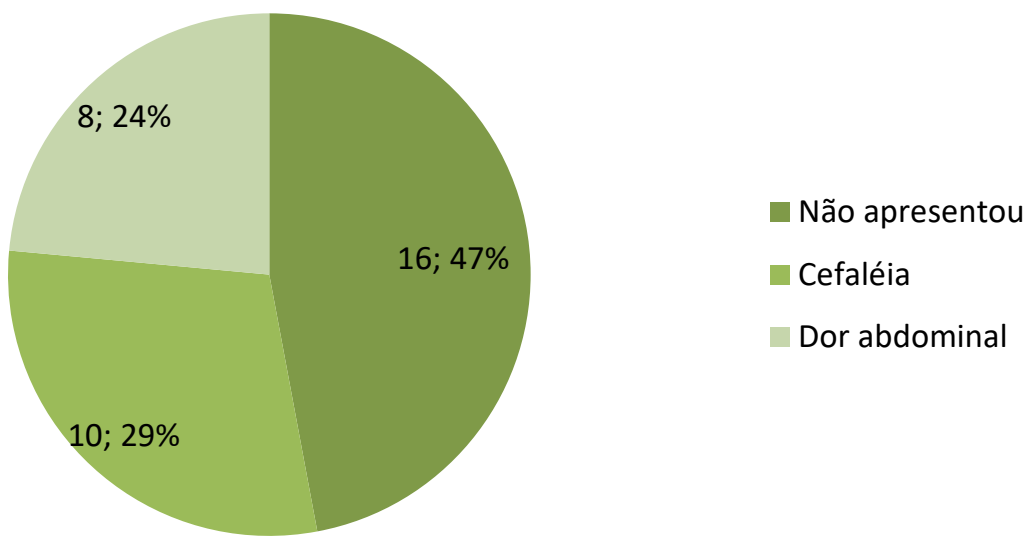

Fonte: Pesquisa de campo, Nova Canaã-BA.

\section{Conclusão}

Pode-se concluir neste estudo que a maioria dos pacientes com HAS entrevistados faz uso de uma combinação de medicamentos anti-hipertensivos para o controle da pressão arterial, pois apresentam alto risco cardiovascular, e que 53\% apresentam alguma queixa relacionada ao uso do medicamento.

O elenco de medicamentos apresentados no estudo está listado na Relação Nacional de Medicamentos Essenciais (RENAME). Em 2004 a Política Nacional de Assistência Farmacêutica em conjunto com a RENAME, melhora as atividades de assistência, restauração, segurança e bem estar dos usuários do Sistema Único de Saúde (SUS), apresentando o medicamento como insumo essencial.

A farmacoterapia da HAS necessita de uso continuo do medicamento para que a terapia 
obtenha sucesso e previna as complicações causadas por ela e isso depende da adesão terapêutica do paciente e da eficiência dos fármacos.

O profissional farmacêutico desempenha um papel de grande importância no combate e prevenção da HAS, pois, ele tem formação especializada sobre o medicamento podendo identificar possíveis interações medicamentosas, classificar os efeitos adversos, orientar e acompanhar o paciente quanto ao uso racional do medicamento garantindo segurança do mesmo.

De acordo com a RDC 44/09, o farmacêutico passa a ser um profissional de saúde habilitado para realizar aferição e monitoramento da pressão arterial e o acompanhamento de pacientes hipertensos, bem como promover ações de educação em saúde para manutenção da adesão ao tratamento e prevenir as complicações cardiovasculares resultantes da HAS.

\section{Referências}

ÁVILA, A.; TAVARES, A.; MACHADO, A. C.; CAMPANA, G. M. E.; LESSA, I.; KRIEGER, E. J.; SCALA, C. L.; NEVES, F. M.; SILVA, G. C. R.; SAMPAIO, R.; FUCHS, S. BRASIL, Ministério da Saúde. VI Diretrizes Brasileiras de Hipertensão. Arquivo Brasileiro de Cardiologia, pág. 1. Rio de Janeiro, 2010. Cap. 1- Conceituação e epidemiologia e prevenção primária. Disponível em: <http://xn--publicaesw3a8m.cardiol.br/consenso/2010/diretriz-hipertens\%C3\%A3o-associados.pdf.> Acesso em: 06/03/2016.

BRASIL, Ministério da Saúde, Secretaria de Ciência Tecnologia e Insumos EstratégicosDepartamento de Assistência Farmacêutica e Insumos Estratégicos. Relação Nacional de Medicamentos Essenciais- RENAME 9a edição, p. 8-10, Brasília, 2015. Disponível em: <http://www.saude.pr.gov.br/arquivos/File/0DAF/RENAME2014ed2015.pdf.> Acesso em: 08/11/2016.

, Ministério da Saúde, Secretaria de Atenção à Saúde, Departamento de Atenção Básica. Caderno de Atenção Básica- Estratégias para o cuidado da pessoa com doença crônica- Hipertensão Arterial Sistêmica, p. 62, Brasília, 2015. Disponível em: <www.google.com.br/webhp?sourceid=chromeinstant\&ion=1\&espv=2\&ie=UTF$8 \# q=$ caderno\%20atencoa\%20basica\%20hipertensao\%202015>. Acesso em 08/11/2016.

BRUNTON, L. L.; CHANNER, B. A.; KNOLLMANM, B. C. As Bases Farmacológicas da Terapêutica de Goodman \& Gilman. 12. Ed. - Porto Alegre, 2012, pag. 672-678.

CARVAlHO, S. A.; FILHO, R. D.; ROBERTO, L. A.; SILVA, C. M. C.; FÓFANO, A. G. A percepção da população sobre a hipertensão arterial sistêmica e seus fatores de riscos. 
Disponível em: <http://www.actabiomedica.com.br/índex php/article/view1108181>. Acesso em:27/05/2016.

KOROLKOVAS, A.; FRANÇA, F. F. A. Dicionário Terapêutico Guanabara. Editora Guanabara Koogan. Rio de Janeiro, 2011.

LOTEMBERG, P. M. E.; GUIMARÁES, C. A.; NEGRÃO, E. C.; FORJAZ, M. L. C.; LOPES, H.; SANTOS, E. J.; ÂNGELO, F. L.; GUSMÃO, L. M.; LIPP, M.; KOHLMANN, B. E. M.; PÓVOA, R.; CARVALHO, T. VI Diretrizes Brasileira de Hipertensão, 2010. Disponível em: <http://www.scielo.br/scielo.php?script=sci_arttext\&pid=S0066-782X2010001700001>. Acesso em: 27/11/2016.

MACHADO, M. C.; PIRES, C. G. S.; LOBÃO, W. M. Concepções dos Hipertensos sobre os fatores de risco para a doença. Escola de Enfermagem. Universidade Federal da Bahia, Salvador, 2010. Disponível em: <http://www.scielosp.org/pdf/csc/v17n5/a30v17n5.pdf.> Acesso em: 08/11/2016.

MARTELli, A.; LONGO, T. A. M.; SERIANI, C.; Aspectos Clínicos e Mecanismo De Ação Das Principais Classes Farmacológicas Usadas No Tratamento Da Hipertensão Arterial Sistêmica. Dissertação (Mestrado). Pontifícia Universidade Católica do Paraná. Paraná, 2008. Disponível em: <file://C:/Users/W\%2010/Downloads/bs-4622.pdf> Acesso em: 07/03/2016.

NOBRE, F.; COELHO, B. E.; LOPES, C. P.; GELEITE, M. J. T. Hipertensão arterial sistêmica primária. Disponível em: < http://www.revistas.usp.br/rmrp/article/view/69136> Acesso em: 27/11/2016.

NOVA CANAÃ | Censo Demográfico 2010. Disponível em: CNEFE - Cadastro... IBGEwww.cidades.ibge.gov.br/.../temas.php?...bahia\%7Cnova-canaa\%7Ccens. Acesso em: 10/03/2016.

NOVA CANAÃ, Prefeitura Municipal de Nova Canaã. Consolidado das famílias Cadastradas do ano de 2015 da zona geral.

NOBRE, F.; COELHO, B. E.; LOPES, C. P.; GELEITE, M. J. T. Hipertensão arterial sistêmica primária. Disponível em: <http://www.revistas.usp.br/rmrp/article/view/69136> Acesso em: 07/03/2016.

OLIVEIRA, P. A. R.; de MENEZES, F. G. Atenção farmacêutica a pacientes hipertensos. Disponível em: <https://revistas.ufg.br/REF/article/view/20622/13789> Acesso em: $18 / 11 / 2016$.

OLIVEIRA, T. S.; OLIVEIRA, L. P. Hipertensão arterial sistêmica em mulheres. Disponível em:<http://www.cpgls.pucgoias.edu.br/8mostra/Artigos/SAUDE\%20E\%20BIOLOGICAS/HI PERTENS\%C3\%830\%20ARTERIAL\%20SIST\%C3\%8AMICA\%20EM\%20MULHERES.p df.> Acessado em: 08/11/2016.

RIBEIRO, B. A.; VIANNA, D.; COELHO, B. E.; BARBOSA, E.; ALMEIDA, A. F.; FEITOSA, G.; MORENO, H.; GUIMARÃES, I. J.; RIBEIRO, P. J.; RAMIREZ, F. A. J.; 
Id on Line Revista Multidisciplinar e de Psicoloqia

Id on Line Multidisciplinary and Psycology Journal

MARTINS, V. F. J.; SANTOS, S. A. R. BRASIL,Ministério da Saúde. VI Diretriz Brasileiras de Hipertensão. Arquivo Brasileiro de Cardiologia, 2010 pag 34. Rio de Janeiro. Cap.6Tratamento Medicamentoso. Disponível em: <http: publicações.Cardiol.br/consenso/2010/diretriz_hipertensão_associados.pdf.> Acesso em: $06 / 03 / 2016$

SANTOS, A. S. M. Z. Hipertensão Arterial- Um Problema de Saúde Pública. Disponível em: $<$ http://bases.bireme.br/cgibin/wxislind.exe/iah/online/?IsisScript=iah/iah.xis\&src=google\&ba se $=$ LILACS\&lang=p\&nextAction=lnk\&exprSearch=621766\&indexSearch=ID >. Acesso em: $10 / 03 / 2016$

TOLEDO, M. M.; RODRIGUES, C. S.; CHIESA, M. A.; Educação em Saúde no Enfrentamento da Hipertensão Arterial: Uma nova Ótica para um Velho Problema. Disponível em: <https:// www.scielo.br/pdf/tce/v16 nº 02/a04v16n2>. Acesso em: 06/03/2016.

Como citar este artigo (Formato ABNT):

COQUEIRO, Jaqueline F.R; Santos, Lucy M. Perfil Farmacológico de Pacientes Hipertensos Cadastrados em uma Unidade de Saúde da Família no Município de Nova Canaã-BA. Id on Line Revista Multidisciplinar e de Psicologia, Julho de 2017, vol.11, n.36, p.52-63. ISSN: 1981-1179.

Recebido: 05.06.2017

Aceito: 14.06 .2017 